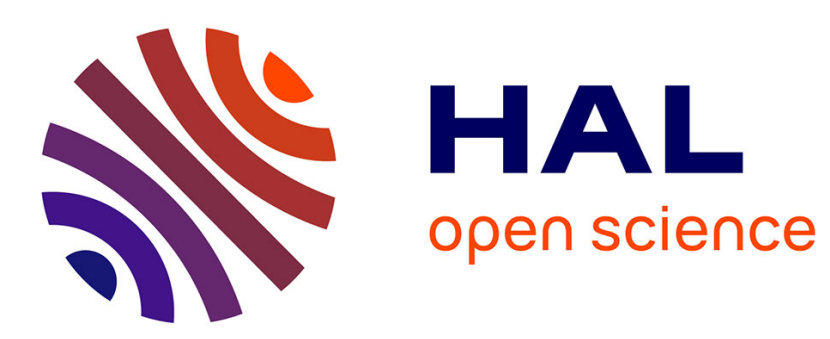

\title{
A finite volume model for multi-component diffusion in magnetically confined plasmas
}

K S C Peerenboom, J van Dijk, W J Goedheer, G Degrez, a M van Der Mullen

\section{To cite this version:}

K S C Peerenboom, J van Dijk, W J Goedheer, G Degrez, a M van Der Mullen. A finite volume model for multi-component diffusion in magnetically confined plasmas. Journal of Physics D: Applied Physics, 2011, 44 (19), pp.194006. 10.1088/0022-3727/44/19/194006 . hal-00615138

\section{HAL Id: hal-00615138 https://hal.science/hal-00615138}

Submitted on 18 Aug 2011

HAL is a multi-disciplinary open access archive for the deposit and dissemination of scientific research documents, whether they are published or not. The documents may come from teaching and research institutions in France or abroad, or from public or private research centers.
L'archive ouverte pluridisciplinaire HAL, est destinée au dépôt et à la diffusion de documents scientifiques de niveau recherche, publiés ou non, émanant des établissements d'enseignement et de recherche français ou étrangers, des laboratoires publics ou privés. 


\title{
A finite volume model for multi-component diffusion in magnetically confined plasmas
}

\author{
K S C Peerenboom ${ }^{1,2}$, J van Dijk ${ }^{1}$, W J Goedheer ${ }^{2}$, G \\ Degrez $^{3,4}$ and J J A M van der Mullen ${ }^{1}$ \\ ${ }^{1}$ Department of Applied Physics, Eindhoven University of Technology, \\ P.O. Box 513, 5600 MB Eindhoven, The Netherlands \\ ${ }^{2}$ FOM Institute for Plasma Physics Rijnhuizen, \\ P.O. Box 1207, 3430 BE Nieuwegein, The Netherlands \\ 3 Von Karman Institute for Fluid Dynamics, \\ 72 Chaussée de Waterloo, B-1640 Rhode-Saint-Genèse, Belgium \\ ${ }^{4}$ Université Libre de Bruxelles, \\ 50 Avenue F.D. Roosevelt, B-1050 Bruxelles, Belgium \\ E-mail: k.s.c.peerenboom@tue.nl
}

\begin{abstract}
In partially ionized, magnetically confined plasmas, the diffusive fluxes of the different species are coupled. Additionally, the fluxes are directionally coupled as well due to the Lorentz force. The challenge in modelling of multicomponent, magnetized plasmas is to take care of this coupling in the numerical method. In this paper, a complex form of the Stefan-Maxwell equations is used, to account for the coupling between the flow directions. To handle the coupling between the species fluxes in the finite volume method, a generalised, coupled form of the exponential scheme is used. The presented numerical method is applied to a magnetically confined hydrogen jet. The results show that the numerical method is capable of describing typical characteristics of magnetized plasmas, such as anisotropic diffusion and the presence of a pressure gradient sustained by the Lorentz force.
\end{abstract}

Submitted to: J. Phys. D: Appl. Phys. 


\section{Introduction}

Magnetically confined, partially ionized plasmas have several interesting applications, such as the plasma source Magnum-PSI, which is used for material research under ITER relevant conditions [1]. To model such plasmas, a multi-component approach is needed, where the effect of the magnetic field on the diffusive fluxes of the different species is taken into account. The diffusive flux of a species in a partially ionized, magnetically confined plasma is not simply a linear function of the gradient of the density or mass fraction; the diffusive fluxes of all the species are coupled. In addition, the flow directions across and around the magnetic field lines are coupled via the Lorentz force.

The coupling between the flow directions across and around the field lines makes it more difficult to calculate the diffusion velocity from the Stefan-Maxwell equations. To overcome this difficulty, we will use a complex from of the Stefan-Maxwell equations, based on the approach presented in $[2,3]$. We will extend the approach of $[2,3]$ to ambipolar diffusion in a magnetic field. To deal with the coupling between the diffusive fluxes of the species in the finite volume approach, we will use the discretization method of [4] where the coupling between the species fluxes is preserved.

The numerical method is tested on an illustrative example of Magnum PSI: an expanding hydrogen plasma. It is shown that the numerical method is capable of describing typical characteristics of Magnum PSI (and other magnetized plasmas), such as anisotropic diffusion and the presence of a pressure gradient sustained by the Lorentz force.

This paper is organised as follows. Section 2 explains how the diffusive fluxes in a magnetized plasma are obtained. In Section 3 these fluxes are discretized with the finite volume method in order to solve the species mass balances. Section 4 presents the model description; the results of the model are given in Section 5. The paper ends with a short conclusions section.

\section{Stefan-Maxwell equations and constraints}

In the presence of a magnetic field $\vec{B}$, the Stefan-Maxwell equations for the diffusive velocity $\overrightarrow{v_{k}}$ of species $k$ can be written as [2]:

$$
\sum_{l} f_{k l}\left(\overrightarrow{v_{k}}-\overrightarrow{v_{l}}\right)-\frac{n_{k} q_{k}}{p} \overrightarrow{v_{k}} \times \vec{B}+\frac{y_{k}}{p} \vec{j} \times \vec{B}=\overrightarrow{d_{k}}
$$

with $f_{k l}$ the mutual friction coefficient between species $k$ and $l, \vec{d}_{k}$ the diffusion driving force, $n_{k}$ the number density, $q_{k}$ the charge, $p$ the pressure, $y_{k}$ the mass fraction and $\vec{j}$ the current density. In the absence of pressure diffusion and thermophoresis, but in the presence of the ambipolar electric field $\vec{E}_{a m b}$, the diffusion driving force is given by:

$$
\overrightarrow{d_{k}}=-\nabla x_{k}+\frac{n_{k} q_{k}}{p} \vec{E}_{a m b}+\frac{n_{k} q_{k}}{p} \vec{v} \times \vec{B}
$$

with $x_{k}$ the mole fraction of species $k$ and $\vec{v}$ the mass averaged velocity of the plasma. The friction coefficients $f_{k l}$ are nonlinear functions of the composition and can be written as:

$$
f_{k l}=\frac{x_{k} x_{l}}{\mathcal{D}_{k l}}, \quad f_{k k} \equiv 0
$$


with $\mathcal{D}_{k l}$ the usual binary diffusion coefficient $[5,6]$. Note that the friction coefficients are symmetric: $f_{k l}=f_{l k}$, and positive: $f_{k l}>0$. Introducing the friction matrix $\mathbf{F}=\left(F_{k l}\right)$, with the elements $F_{k l}$ given by:

$$
F_{k l}= \begin{cases}\sum_{s} f_{k s} & \text { if } k=l, \\ -f_{k l} & \text { if } k \neq l,\end{cases}
$$

the Stefan-Maxwell equations can be rewritten as:

$$
\sum_{l} F_{k l} \overrightarrow{v_{l}}-\frac{n_{k} q_{k}}{p} \overrightarrow{v_{k}} \times \vec{B}+\frac{y_{k}}{p} \vec{j} \times \vec{B}=\overrightarrow{d_{k}}
$$

Note that $\sum_{l} F_{k l}=0$, which demonstrates that $\mathbf{F}$ is singular and hence non-invertible.

\subsection{Along, across and around the field lines}

In the rest of the paper, we will assume that the plasma is cylindrically symmetric and that the magnetic field points in the axial direction. The Stefan-Maxwell equations can then be separated into the directions along, across and around the field lines (Figure 1). In the direction along the field lines $(\|)$, the Stefan-Maxwell equations are

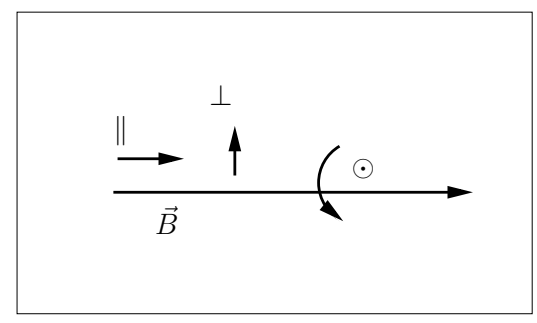

Figure 1. The directions along $(\|)$, across $(\perp)$ and around $(\odot)$ the magnetic field lines.

not influenced by the magnetic field and can be written as:

$$
\sum_{l} F_{k l} v_{l}^{\|}=d_{k}^{\|}
$$

The flow directions across $(\perp)$ and around $(\odot)$ the field lines are coupled:

$$
\begin{aligned}
& \sum_{l} F_{k l} v_{l}^{\perp}-\frac{n_{k} q_{k}}{p} v_{k}^{\odot} B+\frac{y_{k} j^{\odot} B}{p}=d_{k}^{\perp}, \\
& \sum_{l} F_{k l} v_{l}^{\odot}+\frac{n_{k} q_{k}}{p} v_{k}^{\perp} B-\frac{y_{k} j^{\perp} B}{p}=d_{k}^{\odot} .
\end{aligned}
$$

The Stefan-Maxwell equations along the field lines can be solved independently from the other directions. The directions across and around the field lines need to be solved together, which can be done by putting together the $\perp$ and $\odot$ direction in a $2 N \times 2 N$ system, with $N$ the number of species. However, from a computational viewpoint, it is favorable to use complex arithmetic [7]. 
To facilitate coupled solving of the directions across and around the field lines, the Stefan-Maxwell equations are made complex by adding $i$ times equation (8) to equation (7):

$$
\begin{aligned}
& \sum_{l} F_{k l}\left(v_{l}^{\perp}+i v_{l}^{\odot}\right)-\frac{n_{k} q_{k} B}{p}\left(v_{k}^{\odot}-i v_{k}^{\perp}\right)+\frac{y_{k} B}{p}\left(j^{\odot}-i j^{\perp}\right) \\
& =d_{k}^{\perp}+i d_{k}^{\odot} .
\end{aligned}
$$

Substitution of $j^{\perp}=\sum_{k} n_{k} q_{k} v_{k}^{\perp}$ and $j^{\odot}=\sum_{k} n_{k} q_{k} v_{k}^{\odot}$ gives:

$$
\begin{aligned}
& \sum_{l} F_{k l}\left(v_{l}^{\perp}+i v_{l}^{\odot}\right)-\frac{n_{k} q_{k} B}{p}\left(v_{k}^{\odot}-i v_{k}^{\perp}\right)+\frac{y_{k} B}{p} \sum_{l} n_{l} q_{l}\left(v_{k}^{\odot}-i v_{k}^{\perp}\right) \\
& =d_{k}^{\perp}+i d_{k}^{\odot} .
\end{aligned}
$$

When we introduce the complex diffusion velocity $\widetilde{v}_{k}=v_{k}^{\perp}+i v_{k}^{\odot}$, this can be written as:

$$
\sum_{l} F_{k l} \widetilde{v}_{l}+i \frac{n_{k} q_{k}}{p} \widetilde{v}_{k} B-i \frac{y_{k}}{p} \sum_{l} n_{l} q_{l} \widetilde{v}_{l} B=d_{k}^{\perp}+i d_{k}^{\odot}
$$

By adding $\ddagger$ :

$$
-\frac{n_{k} q_{k}}{p} B \sum_{l} y_{l} \widetilde{v}_{l}+y_{k} \frac{\sum_{l} n_{l} q_{l}}{p} B \sum_{l} y_{l} \widetilde{v}_{l}=0,
$$

to each equation, the Stefan-Maxwell equations can be written in matrix vector form as:

$$
\left(\mathbf{F}+i \mathbf{F}^{\prime}\right) \widetilde{\mathbf{v}}=\mathbf{d}^{\perp}+i \mathbf{d}^{\odot},
$$

where the matrix $\mathbf{F}^{\prime}$ is given by $[2,3]$ :

$$
\mathbf{F}^{\prime}=(\mathbf{I}-\mathbf{y} \otimes \mathbf{u}) \mathbf{B}(\mathbf{I}-\mathbf{u} \otimes \mathbf{y}), \quad \mathbf{B}=\operatorname{diag}\left(\frac{n_{i} q_{i} B}{p}\right),
$$

where $\mathbf{u}=(1, \ldots, 1)^{\mathrm{T}}, \mathbf{y}=\left(y_{1}, \ldots, y_{N}\right)^{\mathrm{T}}$, and $\otimes$ denotes the dyadic product of two vectors. The matrix $\mathbf{F}+i \mathbf{F}^{\prime}$ is singular and has nullspace $\mathbf{u}$.

The singularity in $\mathbf{F}+i \mathbf{F}^{\prime}$ can be removed by applying the mass flux constraint $\sum_{k} y_{k} \widetilde{v}_{k}=0$ to the set of Stefan Maxwell equations [8]:

$$
\left(\mathbf{F}+i \mathbf{F}^{\prime}+\alpha \mathbf{y} \otimes \mathbf{y}\right) \widetilde{\mathbf{v}}=\mathbf{d}^{\perp}+i \mathbf{d}^{\odot},
$$

where $\alpha$ is a positive parameter. Inversion of (15) now gives:

$$
\begin{aligned}
\widetilde{\mathbf{v}} & =\left(\mathbf{F}+i \mathbf{F}^{\prime}+\alpha \mathbf{y} \otimes \mathbf{y}\right)^{-1}\left(\mathbf{d}^{\perp}+i \mathbf{d}^{\odot}\right) \\
& =\left(\mathbf{G}+i \mathbf{G}^{\prime}\right)\left(\mathbf{d}^{\perp}+i \mathbf{d}^{\odot}\right) .
\end{aligned}
$$

Note that instead of direct inversion of the Stefan-Maxwell system, it is possible to use iterative methods as discussed for example in [2]. These iterative methods are computationally cheaper than direct inversion. We will not discuss this method here, since in our case the largest computational expense lies in solving the discretized species mass balances (Section 3), and not in the inversion of the Stefan-Maxwell system on each grid point. The real part of the expression (16) gives the diffusion velocity perpendicular to the magnetic field:

$$
\mathbf{v}^{\perp}=\mathbf{G d}^{\perp}-\mathbf{G}^{\prime} \mathbf{d}^{\odot} \text {. }
$$

$\ddagger$ This expression is added to bring the matrix $\mathbf{F}^{\prime}$ to a symmetric form. Since the expression added equals zero, it does not change the diffusion velocities. 
The imaginary part gives the diffusion velocity around the field lines:

$$
\mathbf{v}^{\odot}=\mathbf{G d}^{\odot}+\mathbf{G}^{\prime} \mathbf{d}^{\perp} .
$$

The driving forces still contain the ambipolar field. In the next section, we will explain how the ambipolar field can be eliminated from the equation.

\subsection{Zero-current constraint}

For the direction across and along the field lines, the ambipolar (zero current) constraint is applied. We assume that there is cylindrical symmetry (with the magnetic field pointing in the z-direction). As a consequence, current can flow around the field lines without causing charge separation. The driving forces along, across and around the field lines can thus be written as:

$$
\begin{aligned}
\mathbf{d}^{\|} & =\widetilde{\mathbf{d}}^{\|}+\mathbf{z} \frac{E_{a m b}^{\|}}{p} \\
\mathbf{d}^{\perp} & =\widetilde{\mathbf{d}}^{\perp}+\mathbf{z} \frac{E_{a m b}^{\perp}}{p} \\
\mathbf{d}^{\odot} & =\widetilde{\mathbf{d}}^{\odot}
\end{aligned}
$$

The zero-current constraint for the $\|$ and $\perp$ direction can be written as:

$$
\begin{aligned}
& \left\langle\mathbf{z}, \mathbf{v}^{\|}\right\rangle=0, \\
& \left\langle\mathbf{z}, \mathbf{v}^{\perp}\right\rangle=0,
\end{aligned}
$$

where $\mathbf{z}=\left(n_{1} q_{1}, \ldots, n_{N} q_{N}\right)^{\mathrm{T}}$ and \langle\rangle denotes the inner product in species space. Substitution of the expression for $\mathbf{v}^{\|}$and $\mathbf{v}^{\perp}$ gives:

$$
\begin{aligned}
& \left\langle\mathbf{z}, \mathbf{G}\left(\widetilde{\mathbf{d}}^{\|}+\mathbf{z} \frac{E_{a m b}^{\|}}{p}\right)\right\rangle=0, \\
& \left\langle\mathbf{z}, \mathbf{G}\left(\widetilde{\mathbf{d}}^{\perp}+\mathbf{z} \frac{E_{a m b}^{\perp}}{p}\right)-\mathbf{G}^{\prime} \widetilde{\mathbf{d}}^{\odot}\right\rangle=0,
\end{aligned}
$$

from which we can determine the expression for the ambipolar field:

$$
\begin{aligned}
& \frac{E_{a m b}^{\|}}{p}=-\frac{\left\langle\mathbf{z}, \mathbf{G} \tilde{\mathbf{d}}^{\|}\right\rangle}{\langle\mathbf{z}, \mathbf{G z}\rangle}, \\
& \frac{E_{a m b}^{\perp}}{p}=-\frac{\left\langle\mathbf{z}, \mathbf{G}^{\perp} \tilde{\mathbf{d}}^{\perp}-\mathbf{G}^{\prime} \tilde{\mathbf{d}}^{\odot}\right\rangle}{\langle\mathbf{z}, \mathbf{G z}\rangle} .
\end{aligned}
$$

Backsubstitution of the ambipolar field in the expressions of the velocities across and around the field lines gives:

$$
\begin{aligned}
& \mathbf{v}^{\|}=\left(\mathbf{G}-\frac{\mathbf{G z} \otimes \mathbf{G} \mathbf{z}}{\langle\mathbf{z}, \mathbf{G z}\rangle}\right) \widetilde{\mathbf{d}}^{\|}, \\
& \mathbf{v}^{\perp}=\left(\mathbf{G}-\frac{\mathbf{G} \mathbf{z} \otimes \mathbf{G z}}{\langle\mathbf{z}, \mathbf{G} \mathbf{z}\rangle}\right) \widetilde{\mathbf{d}}^{\perp}-\left(\mathbf{G}^{\prime}-\frac{\mathbf{G} \mathbf{z} \otimes \mathbf{G}^{\prime} \mathbf{z}}{\langle\mathbf{z}, \mathbf{G} \mathbf{z}\rangle}\right) \widetilde{\mathbf{d}}^{\odot}, \\
& \mathbf{v}^{\odot}=\left(\mathbf{G}^{\prime}-\frac{\mathbf{G}^{\prime} \mathbf{z} \otimes \mathbf{G} \mathbf{z}}{\langle\mathbf{z}, \mathbf{G} \mathbf{z}\rangle}\right) \widetilde{\mathbf{d}}^{\perp}+\left(\mathbf{G}+\frac{\mathbf{G}^{\prime} \mathbf{z} \otimes \mathbf{G}^{\prime} \mathbf{z}}{\langle\mathbf{z}, \mathbf{G} \mathbf{z}\rangle}\right) \widetilde{\mathbf{d}}^{\odot} .
\end{aligned}
$$

Note that the matrices relating the diffusion velocities to the driving forces are singular and have nullspace z. This problem was also identified by Giovangigli [9] 
for the case without magnetic field, but in his paper no concrete matrix modifications were proposed. In Appendix A.2, we show appropriate matrix modifications; for a more detailed explanation of these modifications we refer to a future publication [10]. Appendix A explains how the species mass balances are obtained from (23). In the next section, we discuss how the species mass balances are discretized.

\section{Discretization}

As explained in Appendix A, the stationary mass balance for species $k$ in a twodimensional, axisymmetric symmetry can be written as:

$$
\sum_{l}\left(\left(\partial_{\|} U_{k l}^{\|}+\partial_{\perp} U_{k l}^{\perp}\right) y_{l}-\left(\partial_{\|} \Gamma_{k l}^{\|} \partial_{\|}+\partial_{\perp} \Gamma_{k l}^{\perp} \partial_{\perp}\right) y_{l}\right)=S_{k},
$$

with $\mathbf{U}^{\|}=\left(U_{k l}^{\|}\right), \mathbf{U}^{\perp}=\left(U_{k l}^{\perp}\right), \Gamma^{\|}=\left(\Gamma_{k l}^{\|}\right)$and $\Gamma^{\perp}=\left(\Gamma_{k l}^{\perp}\right)$ the convection and diffusion matrices along and across the field lines as derived in Appendix A. It can be seen that the mass balances for all species form a coupled set of convection-diffusion equations. As shown in [4], these can not be discretized using the traditional (scalar) finite volume schemes.

To overcome this problem, a coupled form of the exponential scheme has been introduced $[11,4]$. In this coupled scheme, the solution vectors on the grid points are coupled via discretization matrices instead of discretization coefficients:

$$
\mathbf{A}_{C} \mathbf{y}_{C}=\mathbf{A}_{E} \mathbf{y}_{E}+\mathbf{A}_{W} \mathbf{y}_{W}+\mathbf{A}_{N} \mathbf{y}_{N}+\mathbf{A}_{S} \mathbf{y}_{S}+\mathbf{s}_{C} \Delta x
$$

with $\mathbf{s}_{C}$ the source on the central point $C$ and $\Delta x$ the grid spacing. The discretization coefficients on the central $(C)$, east $(E)$, west $(W), \operatorname{north}(N)$ and $\operatorname{south}(S)$ nodal points are given by:

$$
\begin{array}{ll}
\mathbf{A}_{E}=\frac{1}{\Delta x} \boldsymbol{\Gamma} B\left(\mathbf{P}_{e}\right), & \mathbf{A}_{N}=\frac{1}{\Delta x} \boldsymbol{\Gamma} B\left(\mathbf{P}_{n}\right) \\
\mathbf{A}_{W}=\frac{1}{\Delta x} \boldsymbol{\Gamma} B\left(-\mathbf{P}_{w}\right), & \mathbf{A}_{S}=\frac{1}{\Delta x} \boldsymbol{\Gamma} B\left(-\mathbf{P}_{s}\right) \\
\mathbf{A}_{C}=\mathbf{A}_{E}+\mathbf{A}_{W}+\mathbf{A}_{N}+\mathbf{A}_{S}, &
\end{array}
$$

with $\mathbf{P}$ the Péclet matrix, which is defined as $\mathbf{P}=\Delta x \boldsymbol{\Gamma}^{-1} \mathbf{U}$; the subscripts $e, n, w$ and $s$ denote the interface location where the Péclet matrix is calculated. The Bernoulli function $B()$ is given by:

$$
B(z)=\frac{z}{e^{z}-1} .
$$

Calculation of the of the Bernoulli function of the Péclet matrix is done by diagonalization of the Péclet matrix:

$$
\begin{aligned}
& \mathbf{P} \quad=\mathbf{V} \boldsymbol{\Lambda} \mathbf{V}^{-\mathbf{1}}=\mathbf{V}\left(\begin{array}{ccc}
\lambda_{1} & & \\
& \ddots & \\
& & \lambda_{N}
\end{array}\right) \mathbf{V}^{-1}, \\
& B(\mathbf{P})=\mathbf{V} B(\boldsymbol{\Lambda}) \mathbf{V}^{-\mathbf{1}}=\mathbf{V}\left(\begin{array}{ccc}
B\left(\lambda_{1}\right) & & \\
& \ddots & \\
& & B\left(\lambda_{N}\right)
\end{array}\right) \mathbf{V}^{-1},
\end{aligned}
$$

with $\mathbf{V}$ the matrix with the eigenvectors of $\mathbf{P}$ as its columns and $\boldsymbol{\Lambda}$ a diagonal matrix with the eigenvalues $\lambda_{i}$ of the Péclet matrix. It can thus be seen that the 
coupled scheme boils down to: 1) Decoupling the system by changing to the basis of eigenvectors, 2) Discretization of the decoupled system with the scalar exponential scheme, 3) Transforming back to the original basis.

\section{Model description}

As an illustrative example for Magnum PSI, we will present a two-dimensional convection-diffusion problem for a hydrogen plasma in a magnetic field. Note that the aim of this example is not to give a complete, self-consistent model of Magnum PSI, but to test the ability of the method to describe its characteristic properties. These properties include magnetic confinement of the charged particles and the presence of a radial pressure gradient.

Simulations are performed with the plasma modelling platform Plasimo for different values of the magnetic field. More information on Plasimo can be found in [12]. In the simulations, we solve mass and momentum balances for the plasma bulk and each modelled species individually. The energy balance is not solved, instead we assume a constant temperature of $10000 \mathrm{~K}$ for both the electrons and the heavy particles.

\subsection{Flow field}

The flow field is calculated by solving the mass and momentum balance for the bulk plasma. The momentum balance is given by:

$$
\nabla \cdot(\rho \vec{v} \vec{v})=-\nabla p+\nabla \cdot \overrightarrow{\vec{\tau}}+\vec{j} \times \vec{B}
$$

with $\overrightarrow{\vec{\tau}}$ the viscosity tensor and $\rho$ the mass density which is related to the species densities via:

$$
\rho=\sum_{k} n_{k} m_{k}
$$

The current in the Lorentz force term $\vec{j} \times \vec{B}$ on the right hand side of the momentum balance needs to be calculated from the diffusion velocities. Since we assumed that the plasma is ambipolar and axisymmetric, there are no radial and axial currents, but there is a current in azimuthal direction:

$$
j^{\odot}=\sum_{k} n_{k} q_{k} v_{k}^{\odot}
$$

This azimuthal current with an axial magnetic field gives a radial Lorentz force on the flow. Overall mass conservation is given by:

$$
\nabla \cdot(\rho \vec{v})=0 .
$$

Plasimo uses the SIMPLE algorithm[13] in combination with Karki corrections[14] to solve equations (30) and (33) for compressible flows. Velocities are calculated on a co-located grid. Majumdar interpolation [15] is used to avoid oscillations.

\subsection{Composition}

In the composition calculation, it is assumed that the plasma consists of $H, H^{+}$ and electrons. Chemical reactions are neglected. The mass and momentum (StefanMaxwell eq.) balances are solved as described in Sections 2 and 3. 


\subsection{Geometry and boundary conditions}

The geometry and grid of the model are depicted in figure 2. The geometry is cylindrically symmetric and the magnetic field points in the axial direction. The

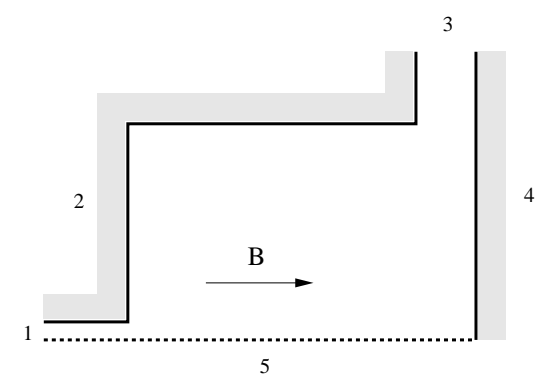

(a) geometry

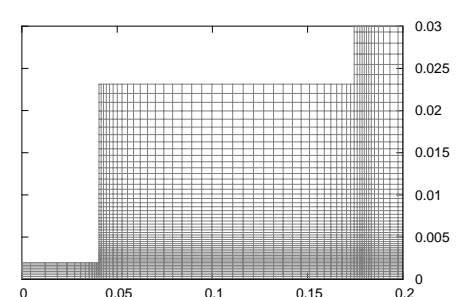

(b) grid

Figure 2. Geometry and grid of the model. 1) inlet of the plasma source, 2) wall, 3) gas exhaust, 4) wall/end plate, 5) symmetry axis.

small cylinder on the left represents the plasma source. The right part of the figure gives the vessel and a gas exhaust. Note that grid stretching is applied in the arc region and the gas exhaust.

At the walls, no-slip boundary conditions are applied to both (radial and axial) velocity components. At the inlet of the arc, and at the gas exhaust, the pressure is prescribed to $15000 \mathrm{~Pa}$ and $1000 \mathrm{~Pa}$ respectively; homogeneous Neumann conditions are applied for the velocity components. At the symmetry axis homogeneous Neumann boundary conditions are applied to both the velocity and the pressure.

At the symmetry axis and gas exhaust, homogeneous Neumann conditions are applied for all species. At the other boundaries the composition is prescribed. In the arc, the mass fraction of $H^{+}$is set to 0.2 , while in the vessel the $H^{+}$mass fraction is set to $1 \mathrm{e}-4$. The mass fractions of the electrons and $H$ at the wall are determined by constraints $\sum_{k} q_{k} y_{k} / m_{k}=0$ and $\sum_{k} y_{k}=1$, respectively.

\section{Results}

The model was run with magnetic strengths of 0,1 and 3 Tesla. The increasing magnetic field strength has the most pronounced effect on the $H^{+}$density, as shown in figure 3. Comparing the simulations with and without magnetic field, it can clearly be seen that radial diffusion at the exit of the plasma source is strongly reduced in the simulations with magnetic field. The simulation at 3 Tesla shows more pronounced magnetic confinement (narrower beam) than the simulation at 1 Tesla. Qualitatively, this is comparable to the experimental results of $[16,17]$. Additionally, at 3 Tesla, a hollow profile may be observed in the centre of the beam.

The degree of magnetization can be expressed in the Hall parameter, which is defined as the ratio between the Larmor frequency and the collision frequency of the electrons:

$$
H=\frac{\Omega_{e}}{\nu_{e}}=\frac{q_{e} B}{m_{e} \nu_{e}},
$$

with $\Omega_{e}$ the Larmor frequency and $\nu_{e}$ the collision frequency. In figure 4 , a radial profile of the Hall parameter, pressure and ambipolar field can be seen. As can be 


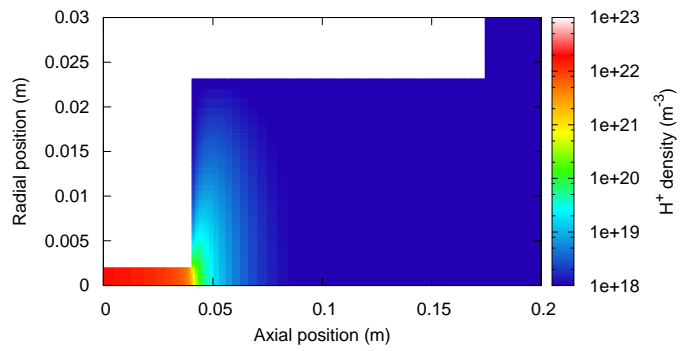

(a) $0 \mathrm{~T}$

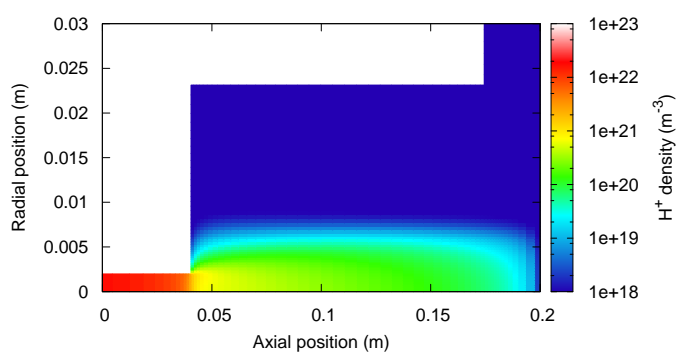

(b) $1 \mathrm{~T}$

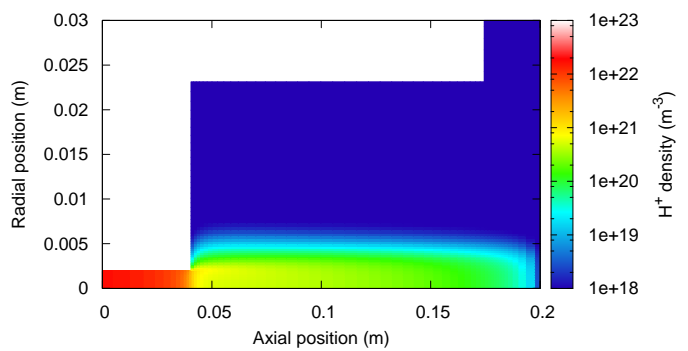

(c) $3 \mathrm{~T}$

Figure 3. $\mathrm{H}^{+}$densities for different values of the magnetic field. The stronger the magnetic field, the narrower (more confined) the plasma beam becomes. In the 3 Tesla simulation, a slightly hollow profile can be observed. 
seen, the Lorentz force on the bulk flow due to the azimuthal current gives rise to a radial pressure gradient. The increased pressure in the centre of the beam increases the collision frequency and thereby reduces the Hall parameter, leading to greater radial diffusion of the ions. We believe this is the mechanism which causes the hollow profile in the ion density, and the flattened profile of the pressure and the Hall parameter.

Whereas in an unmagnetized plasma the ambipolar field points outward, since the ions are the less mobile species, it is negative for magnetized plasmas, where the electrons are the less mobile species. This effect is clearly visible in figure 4 . Due to the hollow profile in the ion density of the 3 Tesla simulation, there is a small region where the ambipolar field is positive.

In figure 5, the axial plots of the pressure and the Hall parameter can be seen. When the plasma is magnetized, the pressure on the axis is larger in the expansion region, due to the earlier mentioned radial Lorentz force. At the inlet of the plasma source where the pressure is high the Hall parameter is low. As the plasma expands the Hall parameter increases, because of the decreasing pressure. 


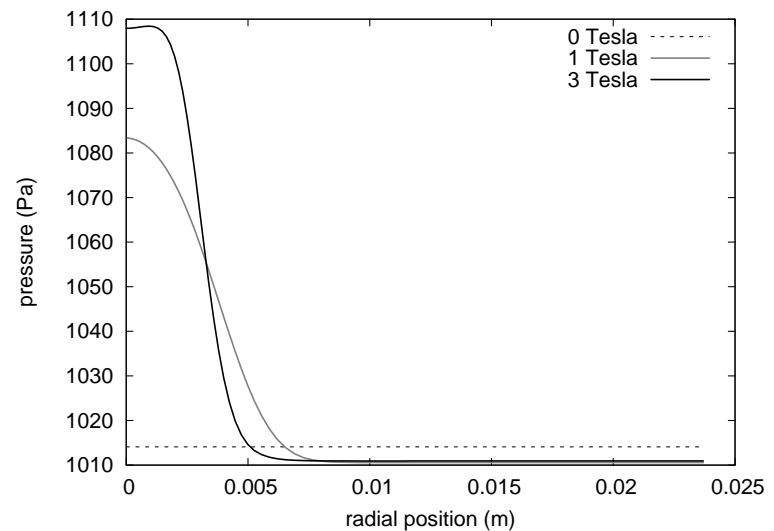

(a) Pressure

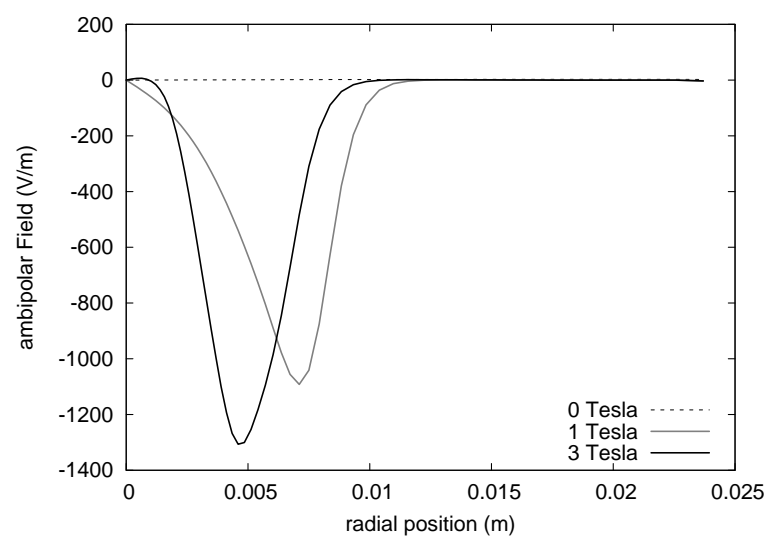

(b) Ambipolar field

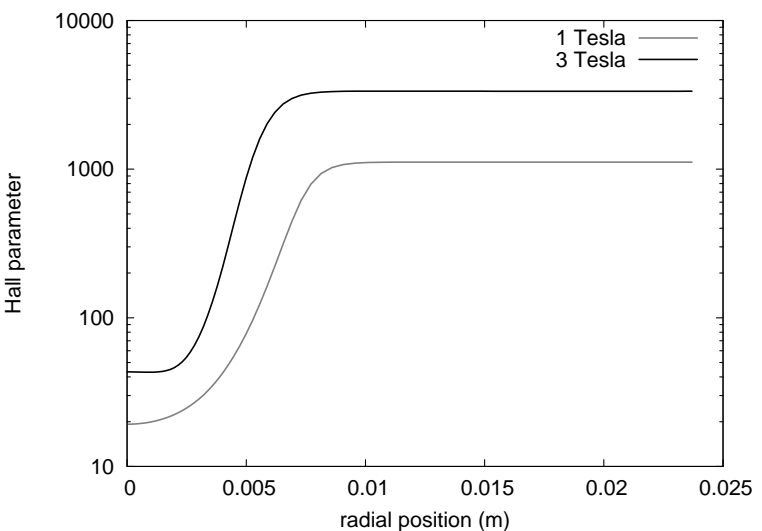

(c) Hall parameter

Figure 4. Radial plots at the axial position $z=0.1$. In the pressure, the ambipolar field as well as in the Hall parameter, the increasing confinement at increasing magnetic fields can be observed. 


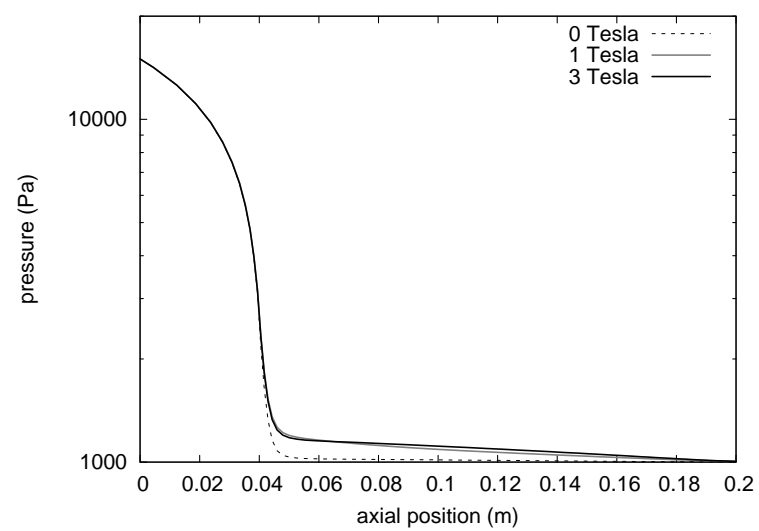

(a) Pressure

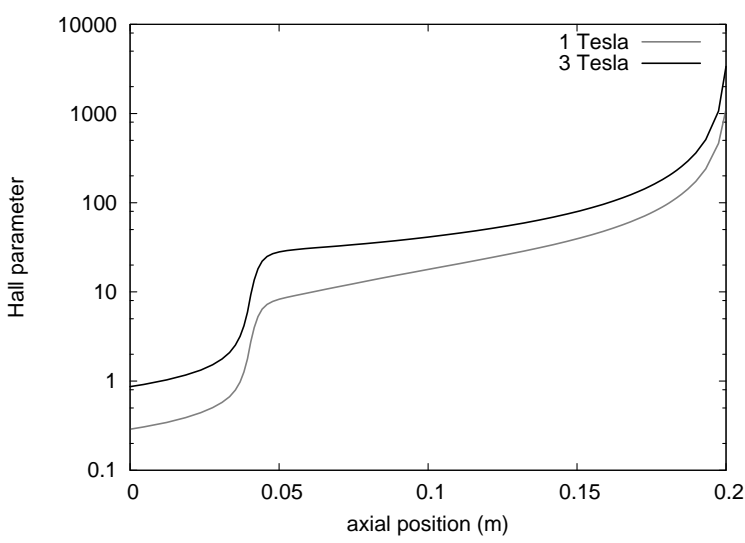

(b) Hall parameter

Figure 5. Axial plots of the pressure and the Hall parameter on the axis. The lower the pressure, the higher the Hall parameter. 


\section{Conclusions}

We discussed a method for the modelling of multi-component, magnetized plasmas with a finite volume scheme. In this method, both the coupling between the flow directions and the species is conserved. The coupling between the flow directions is taken into account by using the Stefan Maxwell equations in complex form. In the discretization, the coupling between the species was taken into account by using a coupled form of the exponential scheme.

As a test case, we used the method to model an expanding hydrogen jet under increasing magnetic field strengths. The results showed expected characteristics of magnetized jets, such as magnetic confinement of the ions, radial pressure gradients and reversal of the ambipolar field.

Although in this paper it was assumed that the plasma is cylindrically symmetric and the magnetic field points in the axial direction, it should, in principle, be possible to apply the method to more general magnetic configurations. Fluxes could be calculated in terms of the directions along, across and around the field lines; after that the fluxes could be transformed to the directions of the cell faces. However, in this transformation, special attention should be paid to the consistency of fluxes at the interface of two adjacent cells, which is of utmost importance in the finite volume method. 
[1] B. de Groot and et al. Magnum-PSI, a plasma generator for plasma-surface interaction research in ITER-like conditions. Fusion Engineering and Design, 74:155-159, 2005.

[2] V. Giovangigli. Transport algorithms for partially ionized mixtures. Journal of Computational Physics, 229:4117-4142, 2010.

[3] V. Giovangigli, B. Graille, T. Magin, and M. Massot. Multicomponent transport in weakly ionized mixtures. Plasma Sources Science and Technology, 19:034002, 2010.

[4] K. Peerenboom, J. van Dijk, J. Ten Thije Boonkkamp, L. Liu, W. Goedheer, and J. van der Mullen. A coupled discretization method for the continuity equations in multi-component mixtures. Submitted to Journal of Computational Physics.

[5] S. Chapman and T. Cowling. The Mathematical Theory of Non-uniform Gases. University Press Cambridge, 1960.

[6] J. Ferziger and H. Kaper. Mathematical Theory of Transport Processes in Gases. North Holland Amsterdam London, 1972.

[7] L.W. Ehrlich. Complex matrix inversion versus real. Communications of the ACM, 13:561-562, 1970 .

[8] V. Giovangigli. Mass conservation and singular multicomponent diffusion algorithms. Impact Comput. Sci. Engrg., 2:73-97, 1990.

[9] V. Giovangigli and B. Graille. Asymptotic stability of equilibrium states for ambipolar plasmas. Mathematical models and methods in applied sciences, 14(9):1361-1399, 2004.

[10] K. Peerenboom et al. On the ambipolar constraint in multi-component diffusion problems. Submitted to Journal of Computational Physics.

[11] J. Ten Thije Boonkkamp, J. van Dijk, L. Liu, and K. Peerenboom. The finite volume complete flux scheme for one-dimensional advection-diffusion-reaction systems. To be published.

[12] J. van Dijk, K. Peerenboom, M. Jimenez, D. Mihailova, and J. van der Mullen. The plasma modelling toolkit plasimo. J. Phys. D: Appl. Phys., 42:194012, 2009.

[13] S. Patankar. Numerical heat transfer and fluid flow. Taylor and Francis, 1980.

[14] K. Karki and S. Patankar. Pressure based calculation procedure for viscous flows at all speeds in arbitrary configurations. AIAA Journal, page 1167, 1989.

[15] S. Majumdar. Role of underrelaxation in momentum interpolation for calculation of flow with nonstaggered grids. Numerical heat transfer, 13:125, 1988.

[16] G. J. van Rooij, H. J. van der Meiden, M.H.J 't Hoen, W. R. Koppers, A. E. Shumack, W. A. J. Vijvers, J. Westerhout, G. M. Wright, and J. Rapp. Thomson scattering at Pilot-PSI and Magnum-PSI. Plasma Physics and Controlled Fusion, 51(12):8, 2009.

[17] A. E. Shumack, V. P. Veremiyenko, D.C. Schram, H. J. Blank, W.J. Goedheer, H. J. van der Meiden, W. A. J. Vijvers, J. Westerhout, N. J. Cardozo, and G. J. van Rooij. Rotation of a strongly magnetized hydrogen plasma column determined from an asymmetric balmer-beta spectral line with two radiating distributions. Physical Review E, 78(4):11, 2008.

\section{Appendix A. The species mass balances}

The stationary mass balance for species $k$ can be written as:

$$
\nabla \cdot\left(\rho \vec{v} y_{k}\right)+\nabla \cdot\left(\rho \overrightarrow{v_{k}} y_{k}\right)=S_{k},
$$

with $\rho$ the mass density, $y_{k}$ the mass fraction of species $k, \vec{v}$ the mass averaged velocity of the plasma, $\overrightarrow{v_{k}}$ the diffusion velocity and $S_{k}$ the mass source term. Rewriting (A.1) in components along and across the field lines gives:

$$
\partial_{\|} \rho v^{\|} y_{k}+\partial_{\|} \rho v_{k}^{\|} y_{k}+\partial_{\perp} \rho v^{\perp} y_{k}+\partial_{\perp} \rho v_{k}^{\perp} y_{k}=S_{k}
$$

In this appendix, we will rewrite the total mass flux to:

$$
\begin{aligned}
& \rho v^{\|} y_{k}+\rho v_{k}^{\|} y_{k}=\sum_{l}\left(U_{k l}^{\|} y_{l}-\Gamma_{k l}^{\|} \partial_{\|} y_{l}\right), \\
& \rho v^{\perp} y_{k}+\rho v_{k}^{\perp} y_{k}=\sum_{l}\left(U_{k l}^{\perp} y_{l}-\Gamma_{k l}^{\perp} \partial_{\perp} y_{l}\right),
\end{aligned}
$$

where $\mathbf{U}^{\|}, \mathbf{U}^{\perp}, \boldsymbol{\Gamma}^{\|}$and $\boldsymbol{\Gamma}^{\perp}$ are the convection and diffusion matrix parallel and perpendicular to the field, respectively. 


\section{Appendix A.1. Conversion to mass fractions}

To be able to solve the species mass balances for the mass fractions $y_{k}$, the diffusion driving forces need to be expressed in the massfractions and the gradients of the mass fractions. Specification of the driving forces gives:

$$
\begin{aligned}
\mathbf{v}^{\|}= & -\left(\mathbf{G}-\frac{\mathbf{G} \mathbf{z} \otimes \mathbf{G} \mathbf{z}}{\langle\mathbf{z}, \mathbf{G} \mathbf{z}\rangle}\right) \partial_{\|} \mathbf{x} \\
\mathbf{v}^{\perp}= & -\left(\mathbf{G}-\frac{\mathbf{G} \mathbf{z} \otimes \mathbf{G} \mathbf{z}}{\langle\mathbf{z}, \mathbf{G} \mathbf{z}\rangle}\right) \partial_{\perp} \mathbf{x}+ \\
& \left(\mathbf{G}-\frac{\mathbf{G} \mathbf{z} \otimes \mathbf{G z}}{\langle\mathbf{z}, \mathbf{G z}\rangle}\right) \frac{v^{\ominus} B}{p} \mathbf{z}+\left(\mathbf{G}^{\prime}-\frac{\mathbf{G} \mathbf{z} \otimes \mathbf{G}^{\prime} \mathbf{z}}{\langle\mathbf{z}, \mathbf{G} \mathbf{z}\rangle}\right) \frac{v^{\perp} B}{p} \mathbf{z} .
\end{aligned}
$$

The gradients of the mole fractions can be converted to the gradients of the mass fractions via a matrix:

$$
\partial_{\|} \mathbf{x}=\widetilde{\mathbf{M}} \partial_{\|} \mathbf{y}, \quad \partial_{\perp} \mathbf{x}=\widetilde{\mathbf{M}} \partial_{\perp} \mathbf{y} .
$$

Special care has been taken to make $\widetilde{\mathbf{M}}$ non-singular [8]. The vector $\mathbf{z}$ can be converted to $\mathbf{y}$ via:

$$
\mathbf{z}=\mathbf{N y}
$$

with $\mathbf{N}=\operatorname{diag}\left(\rho q_{k} / m_{k}\right)$. Note that the driving forces proportional to the gradient of the mass fractions will end up in the diffusion matrix. The forces proportional to the mass fractions will end up in the convection matrix together with the mass averaged velocity.

Appendix A.2. Regularization

In order to keep the diffusion matrix $\boldsymbol{\Gamma}$ non-singular, the matrix $\left(\mathbf{G}-\frac{\mathbf{G z} \otimes \mathbf{G z}}{\langle\mathbf{z}, \mathbf{G z}\rangle}\right)$ should be non-singular. It can, however, be seen that $\mathbf{z}$ is in the nullspace of this matrix. To remove this singularity, the matrices need to be regularized. This can be done by the following modification:

$$
\left(\mathbf{G}-\frac{\mathbf{G z} \otimes \mathbf{G z}}{\langle\mathbf{z}, \mathbf{G z}\rangle}\right)+\beta \mathbf{w} \otimes \mathbf{w},
$$

with $\mathbf{w}=\widetilde{\mathbf{M}}^{-\mathrm{T}} \mathbf{q}$ and $\mathbf{q}=\left(q_{1} / m_{1}, \ldots, q_{N} / m_{N}\right)^{\mathrm{T}}$. The coefficient $\beta$ is a positive parameter.

Appendix A.3. Conversion to mass fluxes

The diffusive velocity vectors $\mathbf{v}^{\|}$and $\mathbf{v}^{\perp}$ can be converted to the diffusive mass fluxes $\mathbf{J}^{\|}$and $\mathbf{J}^{\perp}$ by multiplication with the species mass densities:

$$
\begin{aligned}
& \mathbf{J}^{\|}=\mathbf{R} \mathbf{v}^{\|}, \\
& \mathbf{J}^{\perp}=\mathbf{R} \mathbf{v}^{\perp},
\end{aligned}
$$

with $\mathbf{R}=\operatorname{diag}\left(\rho y_{k}\right)$. 
Appendix A.4. Convection and diffusion matrix

Combination of Sections Appendix A.1, Appendix A.2 and Appendix A.3 gives the following expressions of the convection and diffusion matrices:

$$
\begin{aligned}
\Gamma^{\|} & =\mathbf{R}\left(\mathbf{G}-\frac{\mathbf{G} \mathbf{z} \otimes \mathbf{G z}}{\langle\mathbf{z}, \mathbf{G z}\rangle}+\beta \mathbf{w} \otimes \mathbf{w}\right) \widetilde{\mathbf{M}} \\
\mathbf{\Gamma}^{\perp} & =\mathbf{R}\left(\mathbf{G}-\frac{\mathbf{G z} \otimes \mathbf{G z}}{\langle\mathbf{z}, \mathbf{G z}\rangle}+\beta \mathbf{w} \otimes \mathbf{w}\right) \widetilde{\mathbf{M}} \\
\mathbf{U}^{\|} & =\rho v^{\|} \mathbf{I}, \\
\mathbf{U}^{\perp} & =\rho v^{\perp} \mathbf{I}+\frac{B v^{\odot}}{p} \mathbf{R}\left(\mathbf{G}-\frac{\mathbf{G} \mathbf{z} \otimes \mathbf{G z}}{\langle\mathbf{z}, \mathbf{G} \mathbf{z}\rangle}\right) \mathbf{N} \\
& +\frac{B v^{\perp}}{p} \mathbf{R}\left(\mathbf{G}^{\prime}-\frac{\mathbf{G}^{\prime} \mathbf{z} \otimes \mathbf{G} \mathbf{z}}{\langle\mathbf{z}, \mathbf{G z}\rangle}\right) \mathbf{N} .
\end{aligned}
$$

